\title{
Ulleung-do earthworms - Dagelet Island revisited
}

\author{
Robert J. Blakemore \\ College of Natural Science, Hanyang University, Seoul 133-791, Korea \\ *Correspondent: rob.blakemore@gmail.com
}

\begin{abstract}
Previous surveys on Ulleung-do (Dagelet Isl.) found just ten earthworm taxa while the current survey unearthed a dozen species. Placement of parthenogenetic 'tube' Amynthas dageletensis Hong \& Kim, 2005 is resolved as it clearly belongs in synonymy of Metaphire soulensis (Kobayashi, 1938) which becomes a new Ulleung-do record. Other new megascolecids are recorded since Amynthas heteropodus (Goto \& Hatai, 1898) and A. baemsagolensis Hong \& James, 2001 are confirmed as synonyms of A. corticis (Kinberg, 1867) and A. carnosus (Goto \& Hatai, 1899), respectively. The latter taxon is differentiated and its synonym A. pingi (Stephenson, 1925) provisionally revived. New lumbricids are Bimastos parvus (Eisen, 1874), Eisenia japonica (Michaelsen, 1892) and questionably Dendrodrilus rubidus (Savigny, 1826). All confirmed species are common exotics with no endemics. Drawida moniligastrids were not yet located on this remote island for reasons unknown.
\end{abstract}

Keywords: Island biodiversity, soil fauna, synonyms, terrestrial survey, volcanic activity

(C) 2013 National Institute of Biological Resources

DOI: $10.12651 /$ JSR.2013.2.1.055

\section{INTRODUCTION}

Ulleung-do (울릉도 $37^{\circ} 30^{\prime} \mathrm{N} 130^{\circ} 52^{\prime} \mathrm{E}$ ) also spelt Ulleung-do or Ulreung-do and formerly known to Europeans as Dagelet Isl. or to the Japanese as part of the Takeshima group is a $73.15 \mathrm{~km}^{2}$ breached oceanic stratovolcano about $120 \mathrm{~km}$ East of the South Korean mainland with its highest mountain $984 \mathrm{~m}$ at Seonginbong Peak. Its age has been calculated as only 1.5 million yrs (Kim, 1985), with the last major eruption estimated at ca. 10,000 year ago that covered the island in ignimbrite and tephra and probably destroyed habitats of any resident earthworms. It has been intermittently inhabited over the last few millennia by people who brought plants and seedlings from Korea, China and Japan, latterly during Japanese occupation. However, several endemic plants are also located there (e.g. Acer takesimense Nakai) leading Pfosser et al. (2002) to state:

"On Ullung Island are 37 endemic taxa of angiosperms (Sun and Steussy 1998) with a total flora of approximately 700 species (c. 500 native: Lee and Yang, 1981). Among these endemics, nearly all are single representatives of different genera and families that have apparently arrived and diverged via simple anagenetic change. This island, therefore represents a good system in which to test [evolutionary and speciation] hypotheses...".

Because of such statements it is sometimes hyperboli- cally nicknamed the "Galapagos of Asia". About 63 kinds of birds are reported -25 resident plus 38 migratory species; and about 340 species of insects are recorded on Ulleung-do (Wikipedia, Oct., 2012-http://en.wikipedia.org/ wiki/Ulleungdo).

The first earthworm survey by Song \& Paik (1969) recoded ten earthworm species, and a lesser expedition by Hong \& $\operatorname{Kim}(2005)$ recognized just seven of these, meanwhile Hong \& James (2001) used some of the earlier material to claim A. kimhaeiensis and A. baemsagolensis as new species from "Dagelet I." (amongst other places). Both names are apparent synonyms of A. carnosus (Goto \& Hatai, 1899) along with many others (Kobayashi, 1936; Blakemore, 2012a). Other material was erected as a presumed endemic A. dageletensis Hong \& Kim, 2005 that was considered the same as Metaphire soulensis (Kobayashi, 1938) by Blakemore (2008a: 20, b: 5, 2010b).

These past results are compared to taxonomic revision based on new material in the current paper following a recent brief survey trip by the author on $24-27^{\text {th }}$ September, 2012.

\section{Materials ANd Methods}

The main areas surveyed were: $24^{\text {th }} \& 25^{\text {th }}$ Sept. - Tongumi beside roads and creek-banks near hillside farms; $25^{\text {th }}$ Sept. - bus-stop at accessible summit of Mt Naribun- 
ji then following roads and creeks to the north coast; $26^{\text {th }}$ Sept. - between main towns of Sadong and Dodong; $27^{\text {th }}$ AM Sept. - Arae-tongumi above ferry port where new road has been cut into the hillside.

In order to preserve nature, only mature specimens that were thought representative of known or new taxa from field identifications and by their behaviour were kept for study.

Taxonomic determinations follow the methodology and classifications in Sims \& Easton (1972) and Blakemore (2002; 2010b; 2012d). Specimens in $80 \%$ EtOH are lodged in NIBR facility and small tissue samples sent to Hanyang University (courtesy of Seunghan Lee) for mtDNA COI barcode analysis (data in the Appendix). Genetic analysis via 'MEGA 5' (wwww.megasoftware.net) and BLAST programs (www.blast.ncbi.nlm.nih.gov/BLAST. cgi). Those barcode results that are confidently proven will eventually be uploaded to GenBank (www.blast.ncbi. nlm.nih.gov/genbank) and/or Bold Systems (www. boldsystems.org).

Abbreviations are: GMs - genital markings, lhs - left hand side; ?=uncertainty.

\section{TAxonomic Results}

Results are summarized in Table 1.

\section{Enchytraeidae}

Several of these microdrile worms were noted during the survey but were not kept, as explained in the Jeju study and unnecessary to repeat here (Blakemore, 2013). Objective rationale for researching aquatic or marine worms when crucial terrestrial species are so poorly known and endangered seems unconvincing and difficult to justify without good ecological/economic reason. Without earthworms/soil, dependent species/systems are imperiled.

\section{Megascolecidae}

\section{Amynthas agrestis (Goto \& Hatai, 1899)} [Fig. 1]

Material examined. IV0000261245, mature specimen from Mt Naribunji providing DNA sample w48. IV0000 261245, two mature specimens from Tongumi (sample also contains two sub-adults that may actually be $A$. corticis). IV0000261246, three mature specimens from Tongumi, one a posterior amputee providing DNA sample w49.

Description. Lengths 100-140 mm, segments ca. 105. Light brown dorsum without mid-line, otherwise body grey. Dorsal pores from $11 / 12$ or $12 / 13$. Spermathecal pores 5/6/7/8. Male pores absent. Markings as dark patches on elongate on $7 \&$ paired in 8 in some specimens. Septa 8/9/10 aborted. Holandric, seminal vesicles large in $11 \& 12$. Ovaries in 13, ovisacs absent. Intestine from 15 with manicate caeca from 27. Gregarines present.

Remarks. Specimens conform to earlier descriptions from Japan and to Song \& Paik's (1969: 14, figs. 1, 8 \& $9)$, their Ulleung-do record thus confirmed. Further work on revision of this taxon is currently in progress in Japan as noted by Blakemore (2012c: 106, 117 and 2013).

Note. It was predicted, based on the spermathecae being similar to those of M. hilgendorfi, that forms of this worm with non-superficial male pores may eventually be found requiring its transfer to genus Metaphire. Moreover, male pores were mistakenly reported on penes (e.g. for $P h$.

Table 1. Summary of Ulleung-do earthworms surveys and taxonomic decisions compared

\begin{tabular}{|c|c|c|c|}
\hline No. & Song \& Paik (1969) & Hong \& Kim(2005) & Current study \\
\hline 1 & & & ?Enchytraeus spp. \\
\hline 2 & Pheretima agrestis & Amynthas agrestis & Amynthas agrestis \\
\hline 3 & Pheretima sp. 2 & Amynthas baemsagolensis* & Amynthas carnosus (inc. A. kimhaeiensis \& baemsagolensis) \\
\hline 4 & Pheretima heteropoda & Amynthas heteropodus & Amynthas corticis (inc. A. heteropodus) \\
\hline 5 & Pheretima hupeiensis & Amynthas hupeiensis & Amynthas hupeiensis \\
\hline $6 a$ & Pheretima phaselus (sic) & Amynthas phaselus & Amynthas maculosus/phaselus (inc. A. serratus?) \\
\hline $6 b$ & Pheretima serrata & Amynthas serratus & Ditto \\
\hline 7 & & & ?Amynthas pingi \\
\hline 8 & Pheretima hilgendorfi & Amynthas hilgendorfi & Metaphire hilgendorfi \\
\hline 9 & Pheretima sp. 1 & Amynthas dageletensis & Metaphire soulensis (inc. dageletensis) \\
\hline 10 & $\begin{array}{l}\text { Allolobophora caliginosa } \\
\text { trapezoides }\end{array}$ & Aporrectodea trapezoids (sic) & Aporrectodea trapezoides \\
\hline 11 & & & Bimastos parvus (inc. B. beddardi?) \\
\hline 12 & & & ?Dendrodrilus rubidus \\
\hline 13 & Eisenia foetida & Eisenia fetida & Eisenia fetida (inc. E. andrei, etc.) \\
\hline 14 & & & Eisenia japonica \\
\hline Totals & 10 & 10 & $12-14$ \\
\hline
\end{tabular}

Hong \& Kim (2005) overlook Hong \& James' (2001) A. kimhaeiensis from Ulleung-do that is now a synonym (with many other names) of A. carnosus 

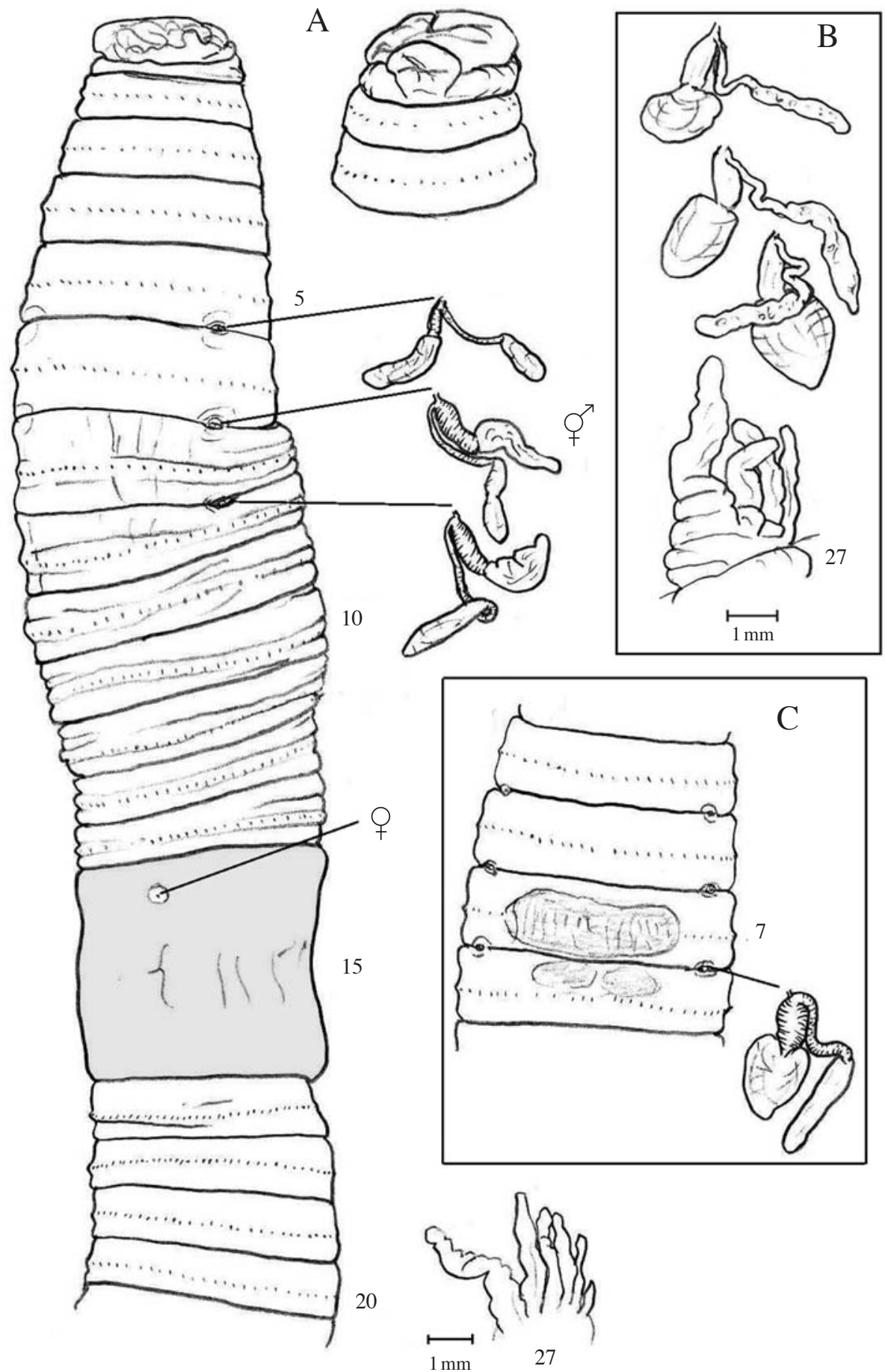

Fig. 1. Amynthas agrestis: A. specimen showing ventral aspect lacking markings, prostomium with spermathecae in situ and caecum in 27; B. specimen from Jeju-do for comparison (Blakemore, 2013); C. second Ulleung-do specimen with patches on 7 \& 8 (indicating amphimixis with concopulants having GMs on 18?). No current specimens have male pores.

striata) or, when Ph. hataii Ohfuchi, 1937 was included in synonymy, slightly invaginated (i.e.,=Metaphire). However in newly inspected material (R.J.B. pers. obs.) from Ibaraki-ken conforming to the type-description the male pores were found to be small and superficial in the setal arc below tumid genital markings (as in Amynthas and as shown by Song \& Paik, 1969: fig. 1) and thus Metaphire hataii was removed from synonymy by Blakemore (2008a) and Amynthas agrestis reinstated (cf. laps in Blakemore, 2013). 


\section{Amynthas carnosus (Goto \& Hatai, 1899)}

Material examined. INV0000261260, ten specimens combined in one sample: two matures from Tongumi, $24^{\text {th }}$ Sept., 2012; two from Naribunji, $25^{\text {th }}$ Sept., 2012; six from Arae-tongumi, $27^{\text {th }}$ Sept, 2012. [INV000261262, an anomalous specimen providing DNA sample w54 removed (see A. pingi below)].

Description. Specimens comply with A. carnosus diagnosis having variable GMs near spermathecal pores in $5 / 6 / 7 / 8 / 9$ and median to male pores that are superficial on 18 in round porophores. [Anomalous specimen INV0 00261262 is provisionally listed under A. pingi].

Remarks. Chinese/Japanese/Korean Amynthas carnosus (Goto \& Hatai, 1899) recently redescribed on the Tokyo museum neotype (Tokyo NSMT An435) by Blakemore (2012a). Synonymy includes: kyamikia Kobayashi, 1934, monstrifera Kobayashi, 1936, sangyeoli, youngtai (with segments miscounted), kimhaeiensis, sinsiensis and baemsagolensis - all Korean names by Hong \& James, 2001, Chinese monsoonus James et al., 2005, fuscus Qiu \& Sun, 2012 plus Amynthas taiwumontis Shen et al., 2013 that was compared to Amynthas fornicatus (Gates, 1935) which, after Chen (1936: 298), is held in synonymy of Chinese A. pingi (Stephenson, 1925) itself usually in synonymy of A. carnosus but provisionally retained pending its review based on the types, as per Blakemore (2012a; 2013) and as noted below. A. taiwumontis was also strangely compared to A. marenzelleri (Cognetti, 1906) that is a long established synonyn of A. corticis (Kinberg) despite its recent redescription by Blakemore (2012c: 114). Note. Song \& Paik (1969: 17, figs. 29-32) described " $P$ eretima sp. 1" from "5 clitellate specimens V 11, 1966, Y.K. Kim" that Hong \& James (2001: 274) named as A. baemsagolensis after its type locality at Mt Jiri on the mainland, including Song \& Paik's material as " 8 clitellate specimens" from "Dagelet I., 5 Aug 1966 (YK Kim)" (sic lapsus?). Hong \& James (2001) only compared $A$. baemsagolensis to their own A. sinsiensis Hong \& James, 2011: 272 - inexplicably overlooking their earlier A. kimhaeiensis Hong \& James, 2001: 270 synonym of A. carnosus - and to A. monstrifera (Kobayashi, 1936) - as indeed had Song \& Paik (1969: 17) - that is itself a synonym of A. carnosus. Hong \& $\operatorname{Kim}(2005: 130,132)$ misspelled the name as "bamsagolensis" (sic lapsus) three times, amongst many other errors (see below) in a paper acknowledging review of all their taxonomic descriptions by $\mathrm{S}$. James.

\section{Amynthas corticis (Kinberg, 1867)} [Fig. 2]

Material examined. IV0000261258, a representative mature specimen from Tongumi (figured); other speci- mens not kept.

Description. $110 \mathrm{~mm}$ long with marking in 7-8-9lhs only and spermathecae in $5 / 6-8 / 9$, i.e., one side complying with "A. heteropodus", one side not. Otherwise agreeing with A. corticis.

Remarks. Part of a cosmopolitan Amynthas corticis species-complex sensu Blakemore (2002; 2010b; 2012d) wherein it is fully described with its many synonyms including Perichaeta heteropoda Goto \& Hatai, 1898 that is still quoted as a valid taxon from Korea by Hong \& Kim (2005) and Hong \& James (2009: 1255). Amynthas sangumburi Hong \& Kim, 2002 is a probable new synonym (Blakemore 2013), provisionally retained as herein pending further research by its original author (Y. Hong pers. comm.).

\section{Amynthas hupeiensis (Michaelsen, 1895)}

Remarks. Common cosmopolitan species, often found in Korea in or near rice paddy fields. Specimens collected

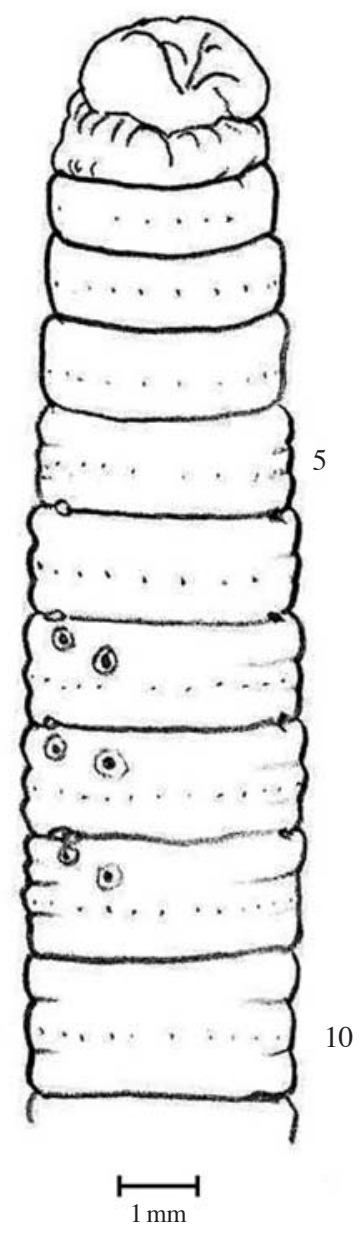

Fig. 2. Amynthas corticis specimen showing asymmetric GMs on anterior ventrum that some authors would have attributed to different taxa on one side compared to the other! 
in current survey were not kept after their field identification based partly on their deep green colour, distinctive smell and coiling behaviour(Blakemore, 2010b).

\section{Amynthas maculosus/phaselus (Hatai, 1930)}

[Fig. 3]

Material examined. INV0000261240, mature specimen from Naribunji providing DNA sample w45. INV00002 61241, two other Naribunji specimens agreeing superficially.

Description. Length $85-110 \mathrm{~mm}$, segments ca. 100. Colour dark on dorsum. First dorsal pore 12/13 (present on clitellum in two of three specimens). Spermathecae in 5/ $6 / 7 / 8$. Male pore in centres of small circular discs or pads on 18. No GMs. Septa aborted in 8/9/10 around gizzard.
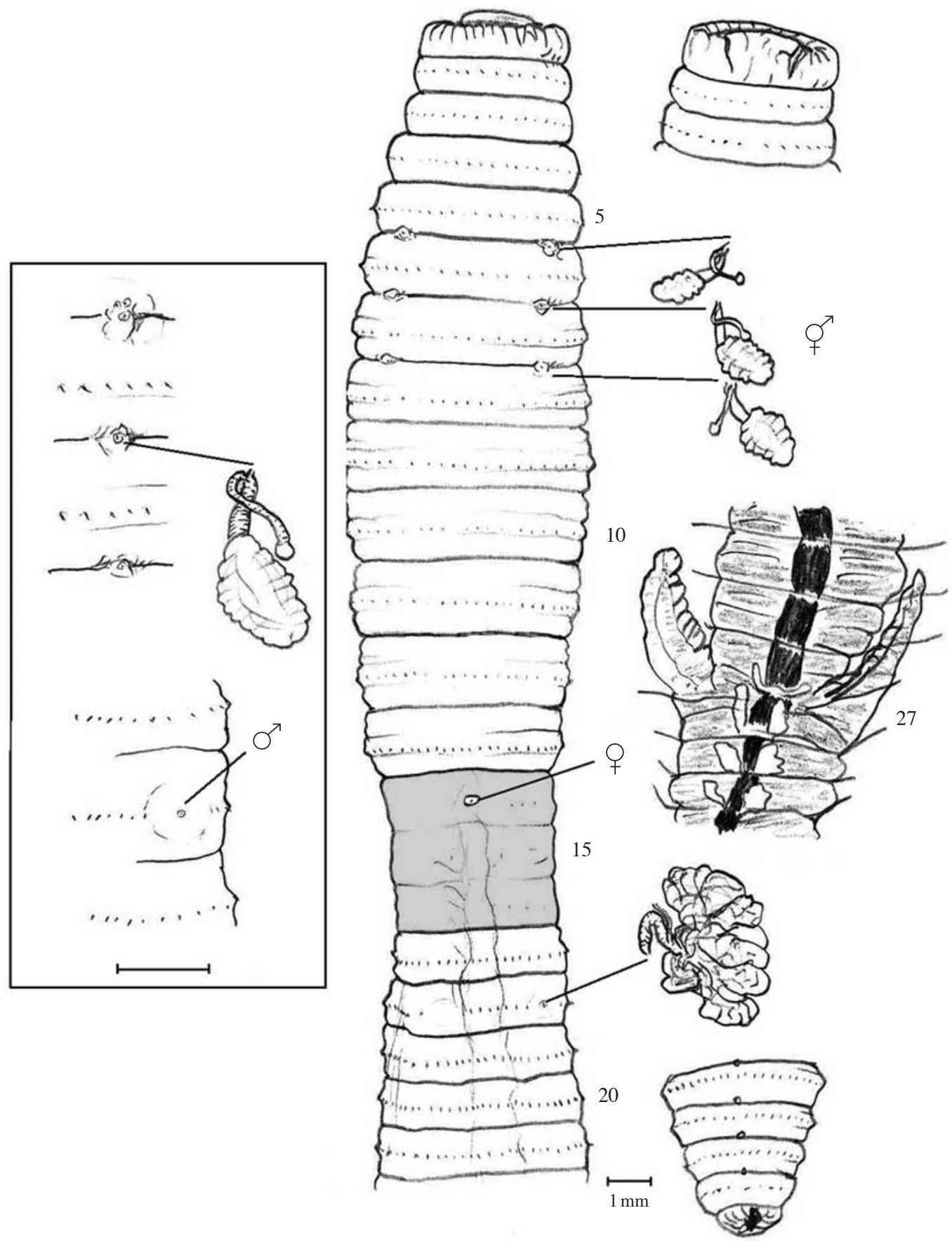

Fig. 3. Amynthas maculosus/phaselus showing ventral aspect, prostomium and pygidium; caeca and reproductive organs in situ; [boxed enlargement of spermathecal and male pore]. 
Spermathecae with corrugated ampullae and clavate diverticula in 6-8. Commissurals in $6 \& 9$, hearts in 10-13. Holandric, testis in sac in 10 , free in 11 ; seminal vesicles in $11 \& 12$ with pseudovesicles in 13 . Ovaries in 13 , no ovisacs. Oesophagus dilated in 12 \& 13, narrow in 14; intestine from 15 with ventrally incised caeca from 27 where dorsal septal glands also commence. Gregarines present.

Remarks. Both Ph. phaselus Hatai, 1930 and Ph. serrata Kobayashi, 1936 were described as present on Ulleung-do by Song \& Paik (1969: 16), this parroted by Hong \& Kim (2005: 131). Since neither seemed to realize that the senior synonym was originally described from Japan, both have: "Distribution: Korea, China". Ph. serrata Kobayashi, 1936 was placed in synonymy of Ph. kamitai Kobayashi, 1934 by Kobayashi (1937: 146), and it was referred to as Ph. phaselus var. kamitai by Kobayashi (1938: 411) and by Song \& Paik (1970: 11) whereas Song \& Paik (1969: 16, figs. 5, 18, $19 \&$ 20) reported it as just $P h$. phaselus (sic) and Song \& Paik (1969, 16, figs. 6, 22-27) attempted to maintain Ph. serrata separately. Blakemore (2013) includes ?Pheretima phaselus tamurai Kobayashi, 1938, Pheretima mutica Chen, 1938 and A. minjae Hong, 2001 in synonymy of A. maculosus (Hatai, 1930) while noting that this taxon requires comparison with $A$. phaselus (in progress). These names require further work after the relationship of A. phaselus (Hatai, 1930) to A. maculosus (Hatai, 1930) is resolved (Blakemore, 2012c; 2012, in prep.) so they are here combined temporarily.

\section{?Amynthas pingi(Stephenson, 1925)}

[Figs. 4, 5]

Material examined. INV000261262, an anomalous Naribunji specimen dissected and providing DNA sample w54 that was, nevertheless, ambiguous (Appendix).

Description. The specimen was slightly larger $(215 \mathrm{~mm})$ with darker brown pigmentation, its spermathecal pores were anterior to intersegments and its GMs are as figured (Fig. 4). It has septal glands pronounced from 15 and appears somewhat intermediate between $A$. carnosus and A. ping $i$ compared to other Ulleung-do specimens that were more like A. carnosus proper.

Remarks. Its DNA data is ambivalent with no close match yet from Korea nor elsewhere (Appendix, Fig. 5) thus it is provisionally placed in a restored $A$. pingi, the next prior synonym of $A$. carnosus. The phylogram shows separation from $A$. carnosus, interestingly, of the two large and darker specimens (w55 \& w56) but it is unlikely that heteroplasmy of mitochondrial DNA in aging cells of older specimens would so greatly affected the result.

This Ullong-do specimen requires comparison with the type of $A$. pingi and with other synonyms in chronologi-

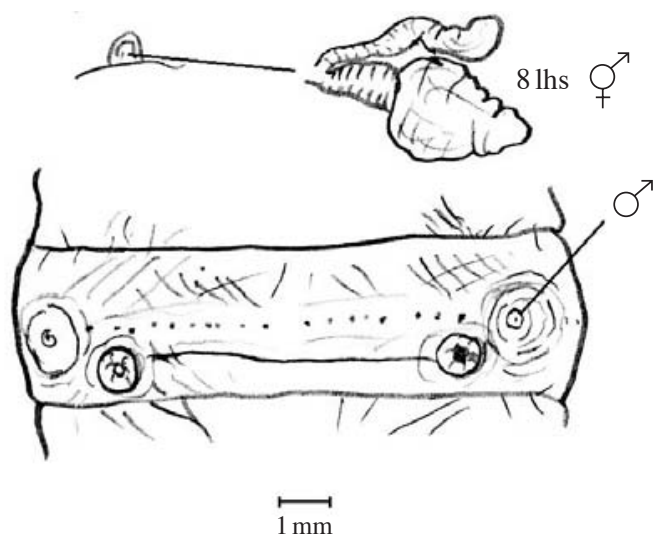

Fig. 4. ?Amynthas pingi specimen showing spermathecal (81hs) and male fields.

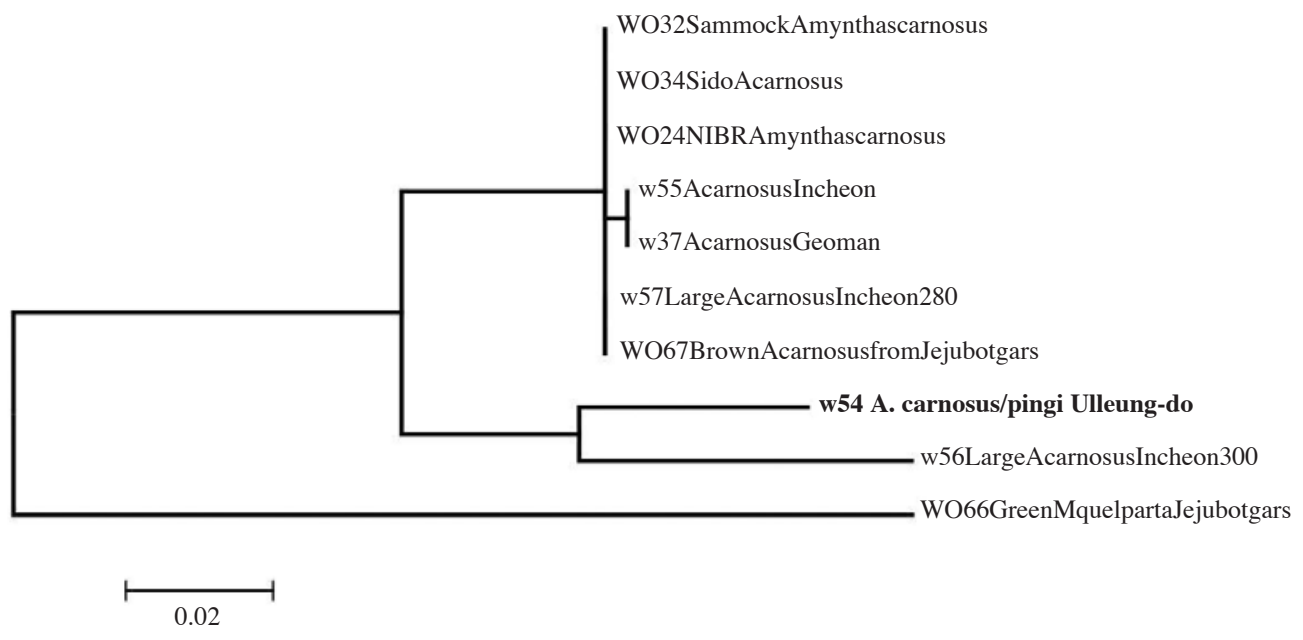

Fig. 5. Phylogram reconstructed by maximum likelihood defaults (via MEGA 5) of mtDNA barcodes for Ulleung-do w54 A. carnosus/pingi compared to other Korean Amynthas carnosus-like material (outgroup is WO66 M. quelparta from Blakemore, 2013: fig. 4). 
cal order. Results of the author's current Korean/Japanese studies are pending and delayed whilst new names seem to be continually added with little consideration of a thorough review nor appreciation of types and priority.
Metaphire hilgendorfi (Michaelsen, 1892)

[Fig. 6]

Material examined. IV0000261247, mature specimen from Tongumi, dissected and figured, providing DNA

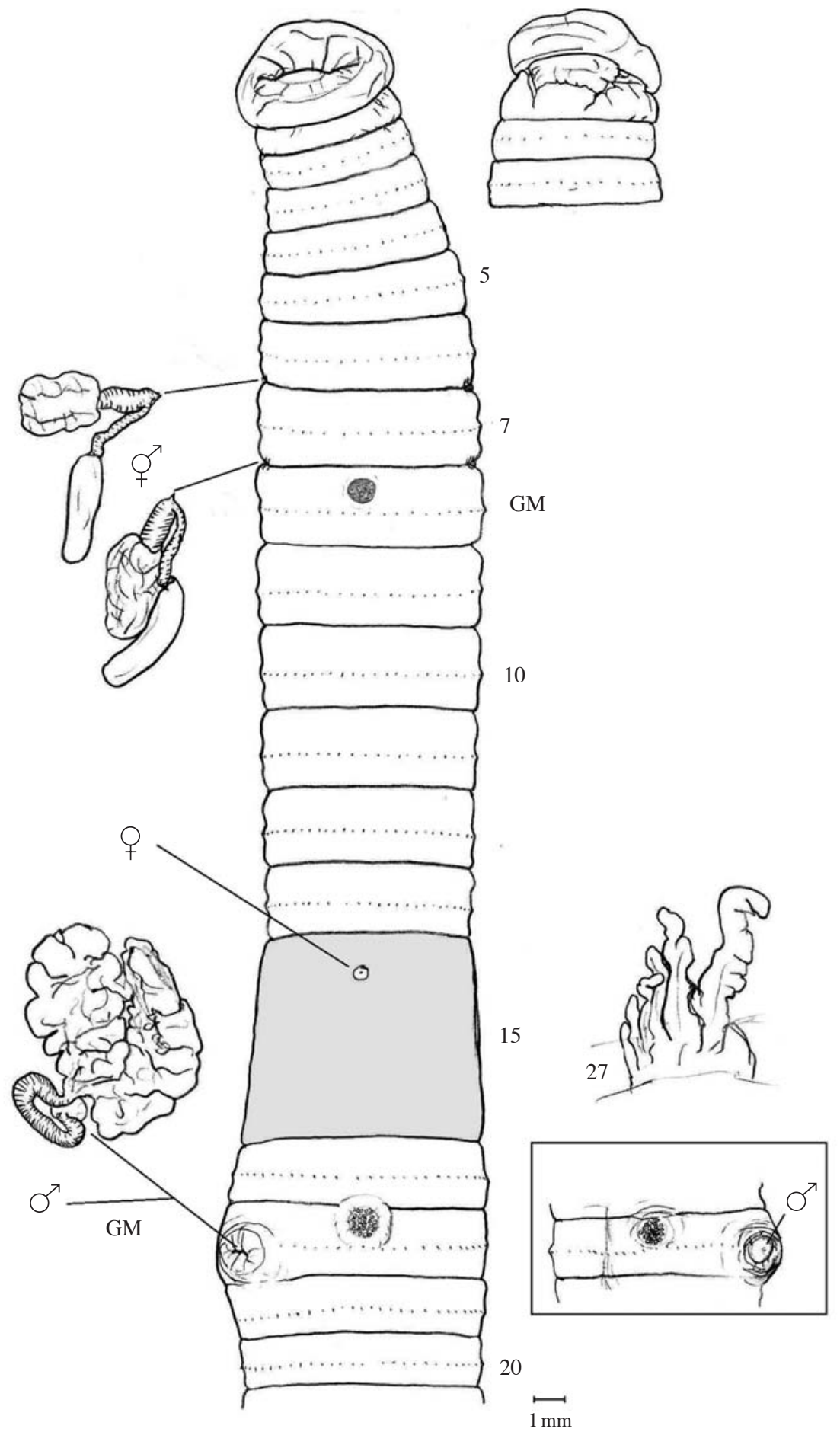

Fig. 6. Metaphire hilgendorfi specimen with invaginated male pore analogue showing ventral aspect, spermathecal and prostate gland in situ, prostomium and manicate caecum; [boxed is a conspecific (and congeneric!) specimen with everted male pore, albeit belonging to Metaphire]. 
sample w50. IV0000261248, a mature specimen from Arae-tongumi (one of several), its male field figured. IV 0000261249, another mature specimen from Tongumi that lacks male pores and GM on 18. Many other specimens released in the field after identification based on size, genital markings and their escape behaviour.

Description. Length $160-170 \mathrm{~mm}$. Segments $\sim 110$. Dorsal pores from 11/12. Spermathecae lateral in 6/7/8. Male pores either absent or present in large invaginated chambers, sometimes everted on blunt penes. GMs circles composed of small discs (up to 20) mid-ventral in 8 and sometimes in 18 too, presetally. Caecae manicate. Many parasites.

Remarks. Despite Blakemore (2003a; 2003b; 2008a) showing that Michaelsen's taxon belonged in Metaphire rather than Amynthas, it was retained in the latter genus by Hong \& Kim (2005) and Hong \& James (2009: 1255). However, Song \& Paik (1969: 15, tab. 1, figs. 3, 13-14, 28) at least, had already shown that twenty-two out of their twenty-three specimens from Dagelet had both a male pore shown to be inverted and with large prostate glands.

Hong \& Kim (2005) make no comment of the state in their 50 specimens, but maintained them in Amynthas presumably on the grounds that the male pores are absent from some specimens or only on one side of the body. Thus presumably one side would belong to genus Amynthas and the other side with male pores would belong to Metaphire under such a scheme (as with A. corticis noted above) which is clearly a ridiculous situation! The current figure agrees with Blakemore (2012c: fig. 3 of "M. glandularis" $=M$. hilgendorfi); see also Blakemore (2002; 2003a; 2003b; 2010a; 2010b; 2012b; 2012c; 2012d) for clarity on correct generic placement of pheretimoids. Review based on Berlin types is in progress by the author.

\section{Metaphire soulensis (Kobayashi, 1938) [Fig. 7]}

Material examined. IV0000261242, a mature specimen from Narabunji, dissected and figured, providing DNA sample w46. INV0000261243, three matures from Narabunji their male fields sketched; INV0000261244, one mature posterior amputee from Do-dong; INV00002612 54, two mature specimens from Tongumi; INV0000261 256, two matures from Arae-tongumi, plus INV0000261 247 another three matures from Arae-tongumi.

Description. Length 75-110 mm with about $100 \mathrm{seg}-$ ments. Colour dark brown dorsum with darker mid-line, clitellum buff 14-16. Dorsal pores from 12/13. No spermathecae, no male pores. GMs are group of one to twelve circular papillae lateral on 18 with glands internally. Septa are aborted in 8/9/10 around gizzard. Hearts in 1013. Holandric, testis small in $10 \& 11$, seminal vesicles in
$11 \& 12$. Pseudovesicles seen in $15 / 16$ and $27 / 28$. Ovaries compact in 13 without ovisacs. Oesophagus dilated in $12 \& 13$, narrow in 15 , intestine in 15 , with manicate caeca from 27; typhlosole not noted. Dorsal septal glands from $28 / 29$.

Remarks. Twenty clitellate specimens were described by Song \& Paik (1969: 17, figs. 7, 33-36) as "Pheretima sp. 2" which was named A. dageletensis sp. nov. based on a Naribunji type by Hong \& Kim (2005: 132, fig. 1) who compared this name to "Amynthas righi Hong \& James, 2001" (sic lapsus for their A. righii) that, nevertheless, appears to actually be separate. However, these specimens all comply with prior $M$. soulensis and its synonyms of Pheretima shinkeiensis Kobayashi, 1938 and Pheretima aokii Ishizuka, 1999 - synonymy as per Blakemore (2003a: 43, b) - plus A. dageletensis Hong et al., 2005 as per Blakemore (2008a; 2008b; 2010) and as confirmed by this study. Its known distribution is Korea and Japan.

\section{Lumbricidae}

\section{Aporrectodea trapezoides (Dugès, 1828)}

Material examined. INV0000261251, one mature specimen and three subadults; plus INV0000261252, eight mature and subadult specimens all from Tongumi.

Remarks. Cosmopolitan species already reported from Ulleung-do by Song \& Paik (1969) and, misspelled as "trapezoids" five times by Hong \& Kim(2005).

\section{Bimastos parvus (Eisen, 1874)}

Material examined. INV0000261250, four specimens from Tongumi, a "parvus" morph providing DNA sample w51, and a "beddardi" morph providing w52. Results in Appendix.

Description. Lengths $42-45 \mathrm{~mm}$. Colour pinkish. Dorsal pores from 4/5. Clitellum in 24-30 lacking tubercular pubertatis (B. parvus sample w51) or clitellum in 25,2631 with tubercula pubertatis in 29-30 (B. beddardi sample w52 that returns DNA result as D. rubidus).

Note. One of the four specimens has first dorsal pore in 6/7 and clitellum in 26,27-31 with faint tubercula in 2930 ; however, segment 16 is abnormal perhaps invalidating these counts.

Remarks. The four specimens looked similar but varied slightly. Bimastos parvus is a cosmopolitan species usually with synonym Bimastos beddardi (Michaelsen, 1894) included. However Hong (2000) - while consistently misspelling parvus as "parva" - attempts to revive this taxon in Korea, possibly following Kobayashi (1941: 156). Nevertheless, this is the first record of either from Ulleungdo. Original orthography, type localities and types of 


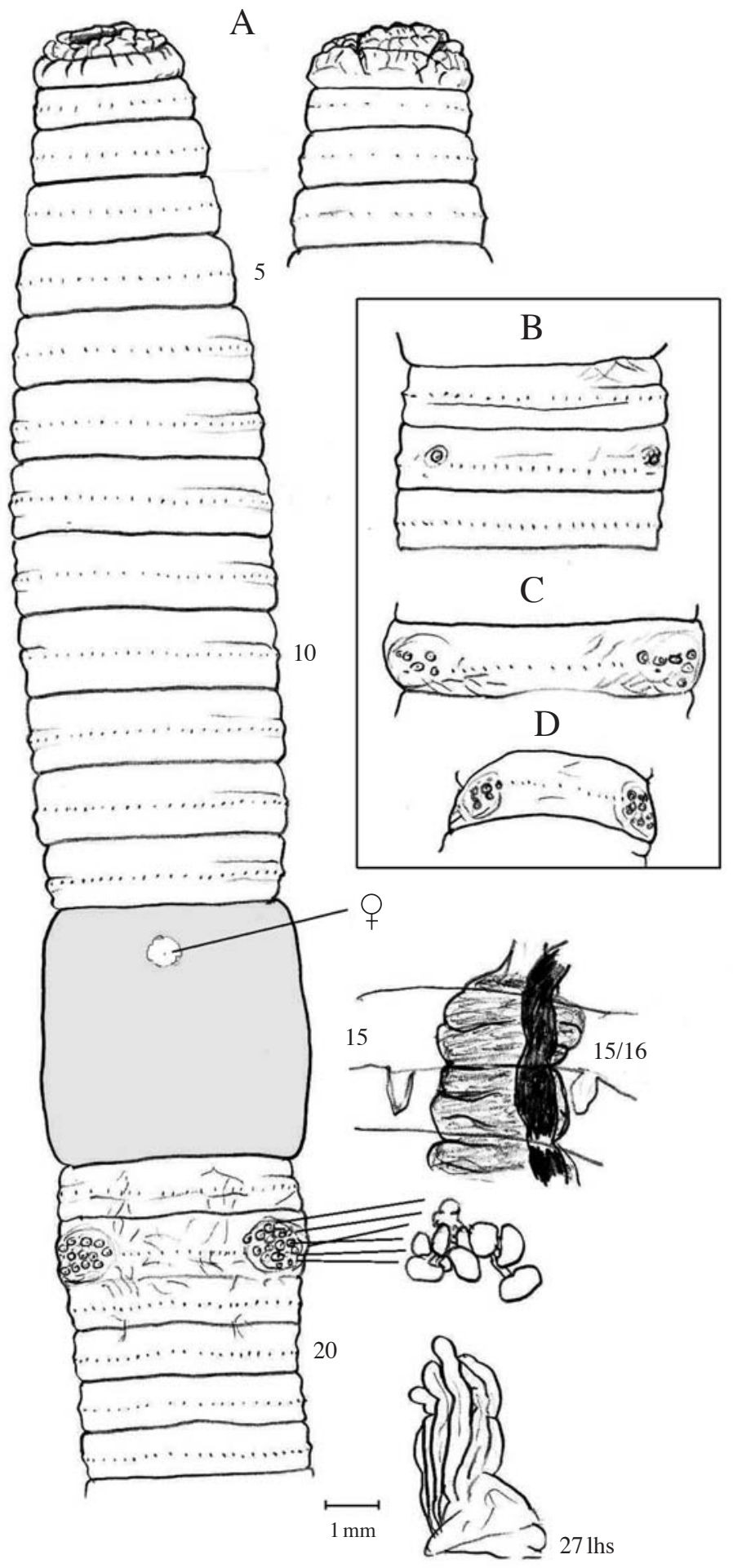

Fig. 7. Metaphire soulensis specimens (all lacking spermathecae and male pores); A. specimen with multi-markings showing ventral aspect; prostomium, 15/16 pseudovesicles, GMs in 18 and manicate caecum; B-D conspecifics encompassing synonyms with fewer GMs.

these two taxa (and an intervening synonym) are: Allolobophora parva Eisen, 1874: 46 [locality Mt Lebanon, New York. Types in US National Museum]. Dendrobaena constricta (part): Friend, 1893: 19 [non
Allolobophora constricta Rosa, 1884: 38 (=Dendrodrilus rubidus)].

Allolobophora beddardi Michaelsen, 1894: 182 [locality Orlando, Orange County, Florida in a rotted tree trunk. 
Type Hamburg, 153]. [Non A. beddardi Ribaucourt, 1896 (=Ap. caliginosaltrapezoides complex)].

\section{Dendrodrilus rubidus species-complex s. Blakemore (2010d)}

\section{Dendrodrilus rubidus (Savigny, 1826)}

Remarks. This is a highly variable and taxonomically intermixed complex comprising four (or more) sub-species with diagnoses overlapping those for Bimastos parvus noted above. Its inclusion in the current list as a new record for Ulleung-do is purely on the basis of the barcode results for "Bimastos beddardi" in the Appendix and therefore it is not confirmed with any confidence. Further DNA data and taxonomic acuity may determine final outcome.

\section{Eisenia fetida (Savigny, 1826)}

Material examined. INV0000261249, four Tongumi specimens, one a posterior amputee.

Description. Lengths 70-85 mm, colour red-striped with pale intersegments and pale laterally on 9-11. Epilobous with first dorsal pore in 4/5. Clitellum 25,26-31,32 with tubercula pubertates in 27,28-30.

Remarks. Cosmopolitan species, previously recorded from Ulleung-do (Song \& Paik 1996: 14, Hong \& Kim, 2005: 130). Often variously mixed with "Eisenia fetida andrei Bouché, 1972" as either a synonym, as invalid morph or 'variety', as a sub-species or as a separate species but none with justification based on types nor priority. For example, Hong et al. (2001) claimed E. andrei from Korea but offered no DNA support. Thus E. andrei is not confirmed from Korea - nor elsewhere - mainly because as there are ca. 15 ignored or overlooked names currently in synonymy of $E$. fetida that have priority over $E$. andrei and are as yet untested. Some of these synonyms, listed in Blakemore (2002; 2004; 2010; 2012d), which are mostly still available under rules of ICZN are:

1. Lumbricus semifasciatus Burmeister, 1835.

2. Lumbricus annularis Templeton, 1836.

3. Lumbricus xanthurus Templeton, 1836 ?

4. Lumbricus olidus Hoffmeister, 1842.

5. Lumbricus luteus Blanchard, 1849.

6. Lumbricus rubrofasciatus Baird, 1873.

7. Allolobophora nordenskiöldi Eisen, 1879 (in part.)? [Review by Blakemore (in press)].

8. Lumbricus annulatus Hutton, 1876/7.

9. Allolobophora foetida var. fimetoria Örley, 1881.

10. Endrilus? annulatus: Smith, 1887.

11. Eisenia nordenskiöldi caucasica Michaelsen, 1903 ?

12. Eisenia foetida attica Tzelepe, 1943.
13. Eisenia fasciata Backlund, 1948 apparently retained as a valid species by some authors, e.g. StöpBowitz (1969: 260, Table V), again this reference mostly overlooked.

\section{Eisenia japonica (Michaelsen, 1892)}

Material examined. INV0000261253, three specimens from Tongumi (plus two pheretimoids in same jar).

Remarks. A new record from Ulleung-do, this species is fully described based on its types by the author in Blakemore \& Grygier (2011).

\section{Discussion}

Compared to islands of similar climate, the earthworm fauna of Ulleung-do is quite impoverished and it supports no endemic earthworms, all species thus far being now recognized as fairly common introduced exotics, shared mainly with Japan. Immediately obvious reasons are its small size and remoteness and, perhaps most significantly, the 'tabula rasa' effect of its recent volcanic activity wiping 'the slate clean'. The rich basaltic soils and oceanic-humid subtropical climate both appear conducive to plants and earthworms, thus the relatively high plant diversity may be attributed to characteristics of plants in new habitats and their possibly unique 'microspecies' facility recognized by botanists. Volcanic Hawaii similarly supports no endemic earthworms (Michaelsen, 1903; Blakemore, 2008c) nor indeed do the Galapagos Islands themselves (Blakemore, 2008d).

Another consideration is that whereas other groups can naturally colonise after transportation via wind and ocean currents, or as seeds attached to birds' feathers, earthworms usually require introduction in soil, most frequently due to human activity (e.g. in potted plants or with tree seedlings).

In contrast, Jeju-do Island, which is substantially larger, closer to the mainland, and presumably has not had such recent volcanic activity, has a current total of around forty earthworms, several appearing endemic (Blakemore, 2013 in current issue). Thus it is assumed that there has been insufficient time to allow introduction of a full complement of soil fauna to Ulleung-do, and that those thus far introduced have either been reinforced by successive introductions or have lower selection pressure. One mystery is why no Drawida have been found thus far as its member species are often small with small cocoons and readily transported but easily overlooked.

Earthworms are not reported from adjacent Juk-do (竹 島) nor from the even more remote Dok-do (독도, 獨島 also known as Liancourt Rocks) that has about 49 plant species, 107 bird species, and 93 insect identified (Wiki- 
pedia - reference http://www.kdi.re.kr/infor/ep_view.jsp ?num=81035). Surveys of these more remote islands and inland areas of Ulleung-do, many of these preserved for military activities, may yield more diverse results.

\section{ACKNOWLEDGEMENTS}

The collection trip was under the auspices of NIBR (Mr TaeSeo Park) with Korean biodiversity funding from Hanyang University (Prof. Wonchoel Lee). Research and reporting was kindly facilitated by managers and staff of NIBR, Incheon and in Yokohama, Japan. JSR editorial advice and efforts of Dr Eun-Young Lee are much appreciated.

\section{REFERENCES}

Blakemore, R.J. 2002. Cosmopolitan earthworms - an eco-taxonomic guide to the peregrine species of the World. VermEcology, PO BOX 414 Kippax, ACT 2615, Australia. Pp. 506 [CD pubication].

Blakemore, R.J. 2003a. Japanese earthworms (Annelida : Oligochaeta): a review and checklist of species. Organisms, Diversity and Evolution 3(3):241-244 [Available from: http://www.urbanfischer.de/journals/ode/].

Blakemore, R.J. 2003b. Ditto. Organisms, Diversity \& Evolution 3(3): Electronic Supplement 2003-11 [Available from: http://www.senckenberg.de/odes/03-11.htm].

Blakemore, R.J. 2004. A provisional list of valid names of Lumbricoidea (Oligochaeta) after Easton, 1983. In: A.G. Moreno and Borges, S. (eds.), Avances en taxonomia de lombrices de tierra/Advances in earthworm taxonomy (Annelida: Oligochaeta). Editorial Complutense, Universidad Complutense, Madrid, Spain. Pp. 75-120.

Blakemore, R.J. 2008a. Japanese earthworms. In: A series of searchable texts on earthworm biodiversity, ecology and systematics from various regions of the World. CD-ROM, Yokohama National University, Japan [Available from: www.annelida.net/earthworm/Japanese\%20Earthworms/ Japanese\% 20Earthworms.pdf].

Blakemore, R.J. 2008b. Korean earthworm species - updated checklist. In: A series of searchable texts on earthworms - Supplemental [Available from: http://www.annelida.net/ earthworm/Korean.pdf January, 2012].

Blakemore, R.J. 2008c. Hawaiian and Puerto Rican earthworm species (original). Op. sit.

Blakemore, R.J. 2008d. Galapagos Islands Earthworms (original). Op. sit.

Blakemore, R.J. 2010a. Saga of Herr Hilgendorf's worms. Zoology in the Middle East 49:7-22.

Blakemore, R.J. 2010b. Cosmopolitan earthworms. $4^{\text {th }}$ Edition. VermEcology, Yokohama, Japan. Pp. 750 [CD publica- tion].

Blakemore, R.J. 2012a. Amynthas carnosus (Goto \& Hatai, 1899) redescribed on its neotype (Oligochaeta: Megadrilacea: Megascolecidae). Journal of Species Research 1(1): 35-43 [Available from: www.nibr.go.kr/download?attach _path=journal\&attach=2012vol1no1_4.pdf].

Blakemore, R.J. 2012b. Restating scope of genus Metaphire Sims \& Easton, 1972: 40 years on (Oligochaeta). Zoology in the Middle East, Supplementum 4:5-14.

Blakemore, R.J. 2012c. On opening a box of worms (Oligochaeta, Megascolecidae) - Historical earthworm specimens transferred to Tokyo from the Saito Ho-on Museum of Natural History in Sendai. Bulletin of the National Science Museum, Tokyo Series A 38(3):95-124.

Blakemore, R.J. 2012d. Cosmopolitan earthworms. $5^{\text {th }}$ Edition. VermEcology, Yokohama, Japan. Pp. 950 [CD publication].

Blakemore, R.J. 2013. Jeju-do earthworms (Oligochaeta: Megadrilacea) - Quelpart Island revisited. Journal of Species Research 2(1):15-54.

Blakemore, R.J. and M.J. Grygier. 2011. Unravelling some Kinki worms (Annelida: Oligochaeta: Megadrili: Lumbricidae) Part III. Journal of Soil Organisms 83(2):231-244.

Chen, Y. 1936. On the terrestrial Oligochaeta from Szechuan II with notes on Gates' types. Contribution of the Biological Laboratory of Science Society of China (Zoology) 11: 269-306.

Hong, Y. 2000. Taxonomic Review of the Family Lumbricidae (Oligochaeta) in Korea. The Korean Journal of Systematic Zoology 16(1):1-13.

Hong, Y. and S.W. James. 2001. Five new earthworms of the genus Amynthas Kinberg (Megascolecidae) with four pairs of spermathecae. Zoological Studies 40(4):269-275.

Hong, Y. and S.W. James. 2009. Some new Megascolecoid earthworms (Oligochaeta). Jounal of Natural History 43: 1229-1256.

Hong, Y. and T.-H. Kim. 2005. The Earthworm fauna from Ulleungdo Island, Korea with one new species of the genus Amynthas (Oligochaeta: Megascolecidae). The Korean Journal of Systematic Zoology 21(2):129-136.

Hong, Y., T.H. Kim \& Y.-E. Na. 2001. Identity of two earthworms used in vermiculture and vermicomposting in Korea: Eisenia andrei and Perionyx excavatus. Korean Journal of Soil Zoology 17:185-190.

Kobayashi, S. 1934. Three new Korean earthworms belonging to the genus Pheretima, together with the wider range of the distribution of Pheretima hilgendorfi (Michaelsen). Journal of Chosen Natural History Society 19:1-11.

Kobayashi, S. 1936. Distribution and some external characteristics of Pheretima (Ph.) carnosa (Goto et Hatai) from Korea. Science Report of the Tohoku Imperial University 11(1):115-138.

Kobayashi, S. 1938. Earthworms of Korea I. Science Report of the Tohoku Imperial University 13(2):89-170. 
Kobayashi, S. 1941. Earthworms of Korea. II. Science Report of the Tohoku Imperial University 16:147-156.

Kim, Y.K. 1985. Petrology of Ulreung volcanic island, Korea - Part 1. Geology. Journal of the Japanese Asociation of Mineralogists, Petrologists and Economic Geologists 80: 128-135.

Michaelsen, W. 1903. Die geographische verbreitung der oligochaeten. Friedländer \& Sohn, Berlin. Pp. 186.

Pfosser, M.F., J. Guzy-Wrobelska, B.-Y. Sun, T.F. Stuessy, T. Sugawara and N. Fujii. 2002. The origin of species of Acer (Sapindaceae) endemic to Ullung Island, Korea. Systematic Botany 27:351-367 [Available from: http:// www. jstor.org/stable/ 3093876].

Sims, R.W. and E.G. Easton. 1972. A numerical revision of the earthworm genus Pheretima auct. (Megascolecidae:
Oligochaeta) with the recognition of new genera and an appendix on the earthworms collected by the Royal Society North Borneo Expedition. Biological Journal of the Linnean Society 4:169-268.

Song, M.-J. and K.-Y. Paik. 1969. Preliminary survey of the earthworms from Dagelet Isl., Korea. Korean Journal of Zoology 12(1):13-21.

Song, M.-J. and K.-Y. Paik. 1970. Earthworms from Chejoodo Island, Korea. Korean Journal of Zoology 13(1):9-14.

Stöp-Bowitz, C. 1969. A contribution to our knowlege of the systematics and zoogeography of Norwegian earthworms. (Annelida, Oligochaeta: Lumbricidae). Nytt magasin for zoologi, Oslo 17:169-280.

Submitted: January 22, 2013, Accepted: February 19, 2013 
Appendix - mtDNA CO1 barcode gene preliminary results.

$>$ w45 Amynthas cf phaselus Ulleung-do

TTTATACTTCATTTTAGGTATTTGAGCTGGAATAATTGGGGCTGGGATAAGCCTTCTTATTCGAATCGAACTAAGACAACCTG GACCATTTTTAGGCAGAGATCAACTATATAATACAATTGTTACTGCTCATGCATTCTTAATAATTTTTTTTCTAGTTATACCA GTATTTATTGGTGGATTTGGAAATTGACTACTACCACTAATACTAGGAACTCCAGATATGGCATTTCCACGACTTAATAATA TAAGATTCTGACTTCTACCCCCATCACTAATTCTATTAGTATCATCTGCTGCAGTAGAAAAAGGTGCTGGAACAGGATGAAC AGTATATCCACCACTAGCAAGAAATATTGCACATGCAGGACCTTCAGTAGACCTTGCAATTTTTTCACTCCATCTTGCTGGTG CATCCTCAATTTTAGGAGCTATTAACTTTATTACTACCGTAATTAATATGCGATGATCTGGGCTTCGCTTAGAACGAATTCCA CTATTTGTCTGAGCGGTAGTAATTACAGTTGTACTACTACTTCTTTCACTTCCAGTTCTTGCAGGAGCCATTACAATACTACT AACAGACCGAAACCTTAACACATCATTCTTCGACCCCGCAGGTGGGGGAGATCCTATTCTATACCAACACCTGTTT Blast result: Amynthas phaselus (AB542519 \& AB542520 from Japan) $=636 / 637(99.9 \%)$.

BLAST w45 vs. w27 (A. kamitai (=maculosus?) from Jeju) Identities=524/618 (85\%), i.e., different species (cf. Blakemore, 2013).

>w46 A. soulensis Ulleung-do many gm - nil result.

> w47 A. soulensis Ulleung-do few gm

TATATTTTATTTTAGGAATTTGAGCTGGAATAATTGGAGCAGGAATAAGACTACTTATTCGAATTGAACTAAGACAGCCTGG CTCTTTCCTGGGAAGAGATCAACTTTATAATACAATCGTAACTGCCCATGCATTTCTAATAATTTTTTTTCTCGTAATACCTGT ATTTATTGGGGGATTCGGAAATTGATTATTACCTCTTATACTAGGTGCACCCGATATAGCTTTCCCCCGACTTAATAATATAA GATTCTGGTTGCTCCCTCCTTCCCTTATTTTATTAGTATCCTCAGCAGCCGTAGAAAAAGGTGCTGGAACAGGATGAACAGT ATACCCGCCACTTGCAAGGAATATTGCTCATGCTGGGCCCTCAGTAGACCTGGCAATTTTTTCCCTCCATTTAGCTGGTGCAT CATCAATTTTAGGAGCAATTAACTTTATTACCACAGTAATTAATATACGATGGTCTGGGCTACGCTTAGAACGAATTCCCCT ATTTGTATGAGCCGTTGTAATTACTGTAGTTCTCCTGCTCCTATCCTTACCTGTCCTAGCCGGTGCTATTACAATACTACTAAC AGATCGAAACCTAAATACATCATTCTTCGACCCAGCAGGGGGTGGGGACCCTATTTTATATCAACATTTATTT

Blast result: Metaphire soulensis (AB542665.1 from Japan)=633/637 (99.4\%), i.e., tolerably agrees but the Japanese identity not certain.

> w48 Amynthas agrestis Ulleung-do no GMs.

CTATACTTTATTTTAGGAATTTGAGCAGGGATAATTGGAGCAGGAATAAGACTCCTAATTCGAATTGAACTAAGACAACCGG GCTCTTTTCTAGGAAGTGACCAACTATATAACACAATTGTAACTGCCCATGCATTTCTAATAATTTTTTTTCTAGTAATACCA GTATTTATTGGTGGATTTGGAAATTGACTATTACCACTTATACTGGGTGCACCAGACATAGCTTTTCCACGACTCAATAATAT AAGATTCTGATTACTTCCCCCATCTCTTATTTTATTAGTGTCCTCGGCAGCCGTAGAAAAGGGTGCGGGAACAGGATGAACA GTATATCCACCACTTGCAAGAAATATTGCACATGCTGGACCATCCGTAGACCTAGCAATTTTTTCTCTTCATCTGGCTGGTGC ATCTTCAATTTTAGGTGCTATTAACTTTATCACCACCGTAATTAATATACGATGATCCGGATTACGTTTAGAACGAATTCCTC TATTTGTGTGGGCAGTTGTAATCACTGTAGTTCTCCTACTTCTATCTCTACCTGTTCTGGCTGGTGCGATTACAATACTCCTAA CAGATCGAAATCTAAATACATCATTCTTTGATCCTGCAGGAGGAGGAGATCCTATTTTATACCAACACTTATTC

Blast result: "Metaphire agrestis" (AB542601 from Japan), 635/637=(99.7\%), i.e., tolerably agrees but the Japanese identity not certain.

>w49 Amynthas agrestis Ulleung-do with pads on 7 \& 8.

TATACTTTATTTTAGGAATTTGAGCAGGGATAATTGGAGCAGGAATAAGACTCCTAATTCGAATTGAACTAAGACAACCGG GCTCTTTTCTAGGAAGTGACCAACTATATAACACAATTGTAACTGCCCATGCATTTCTAATAATTTTTTTTCTAGTAATACCA GTATTTATTGGTGGATTTGGAAATTGACTATTACCACTTATACTGGGTGCACCAGACATAGCTTTTCCACGACTCAATAATAT AAGATTCTGATTACTTCCCCCATCTCTTATTTTATTAGTGTCCTCGGCAGCCGTAGAAAAGGGTGCGGGAACAGGATGAACA GTATATCCACCACTTGCAAGAAATATTGCACATGCTGGACCATCCGTAGACCTAGCAATTTTTTCTCTTCATCTGGCTGGTGC ATCTTCAATTTTAGGTGCTATTAACTTTATCACCACCGTAATTAATATACGATGATCCGGATTACGTTTAGAACGAATTCCTC TATTTGTGTGGGCAGTTGTAATCACTGTAGTTCTCCTACTTCTATCTCTACCTGTTCTGGCTGGTGCGATTACAATACTCCTAA CAGATCGAAATCTAAATACATCATTCTTTGATCCTGCAGGAGGAGGAGATCCTATTTTATACCAACACTTATTC BLAST w48 vs. w49 Identities $=653 / 653(100 \%)$, i.e, ditto $w 48$.

$>$ w50 M. hilgendorfi Ulleung-do - nil result.

$>$ w51 Bimastos parvus Ulleung-do - nil result.

$>$ w52 Bimastos cf. beddardi Ulleung-do

GGGAGCCTTTCTAGGAAGAGACCAACTTTATAATACAATTGTAACAGCCCATGCATTTGTAATAATCTTCTTTCTAGTTATGC CCGTATTTATTGGAGGGTTTGGAAATTGACTTCTCCCTTTAATACTAGGTGCTCCTGATATAGCATTTCCCCGTCTAAATAAC ATAAGATTTTGACTACTACCCCCTGCACTTATTCTCTTAGTATCTTCTGCTGCAGTAGAAAAGGGGGCTGGGACCGGGTGAA CTGTTTACCCCCCTTTATCCAGAAACATTGCGCATGCTGGACCCTCTGTAGATCTAGCAATTTTCTCTCTTCATTTAGCGGGG GCATCCTCAATTCTCGGTGCCATTAACTTTATTACAACAGTTATTAATATACGATGAAGGGGCCTACGTCTAGAACGTATTCC CTTATTTGTATGAGCAGTATTAATTACTGTAGTGTTACTTCTTCTCTCTTTACCAGTTCTTGCTGGCGCAATTACTATACTTCT TACAGACCGAAACCTAAACACCTCATTCTTTGATCCTG

Blast result: Dendrodrilus rubidus (DQ092900, from Austria and JN869911 from Innsbruck) 531/536= (99.1\%), i.e., within usual species boundaries but Austrian identities not confirmed.

> w54 A carnosus/pingi Ulleung-do

TTATACTTTATCTTAGGAATCTGAGCCGGAATAATTGGTGCTGGTATAAGACTTCTTATTCGAATTGAGCTCAGACAACCAG 
Appendix - Continued.

GATCCTTTCTGGGAAGAGATCAACTATATAACACAATTGTAACAGCACACGCATTCCTGATAATTTTCTTTCTAGTTATACCC GTATTCATTGGGGGATTTGGAAATTGACTACTACCACTTATACTAGGAACACCGGATATAGCATTTCCGCGCCTAAACAATA TGAGATTTTGACTATTACCACCATCACTAATCTTACTAGTCTCTTCAGCCGCAGTAGAGAAAGGAGCGGGGACAGGATGGAC AGTATATCCCCCCCTAGCAAGAAATATTGCTCATGCAGGGCCATCAGTAGACCTAGCAATTTTCTCACTCCACTTAGCTGGA GCATCCTCAATTTTAGGGGCCATTAACTTCATTACTACAGTAATTAATATGCGCTGGTCTGGACTACGCCTAGAGCGAATTC CACTATTTGTGTGAGCTGTAGTAATTACTGTGGTGCTACTATTACTATCGTTACCAGTTCTTGCCGGAGCTATTACAATACTA CTAACAGACCGAAACTTAAATACATCATTCTTCGACCCAGCGGGAGGGGGAGACCCAATTTTATACCAACATCTATTT

megaBlast result: Amynthas carnosus (AB543184 from Japan) 593/639 (93\%), i.e., somewhat different to expected result, but Japanese identities unconfirmed.

BLAST w54 vs. w56 (author's large $300 \mathrm{~mm}$ A. carnosus/pingi from Incheon) Identities $=607 / 652(93 \%)$, i.e., this the closest, yet with some difference detected in this species-complex requiring revision based on types and priority (Fig. 5).

BLAST w54 vs. WO67 A. carnosus from Jeju botanic gardens, Identities=605/654 (93\%)(cf. Blakemore, 2013). 